\title{
Optically tunable and rewritable diffraction grating with photoaligned liquid crystals
}

\author{
J. Sun, A. K. Srivastava, ${ }^{*}$ L. Wang, V. G. Chigrinov, and H. S. Kwok \\ Department of Electronic and Computer Engineering, Hong Kong University of Science and Technology, \\ Clear Water Bay, Kowloon, Hong Kong, China \\ *Corresponding author: abhishek_srivastava_lu@yahoo.co.in
}

Received January 28, 2013; revised May 31, 2013; accepted June 3, 2013; posted June 5, 2013 (Doc. ID 184284); published June 27, 2013

\begin{abstract}
An optically tunable and rewritable liquid crystal (LC) diffraction grating cell has been revealed that consists of an optically active and an optically passive alignment layer. The grating profile is created by confining the LC director distribution in alternate planar and twisted alignment domains by means of photoalignment of the LCs. The proposed grating is optically tunable for diffractive and nondiffractive states with a small response time that depends on the exposure energy and LC parameters. In addition, the grating can be erased and rewritten for different diffracting characteristics. These optically tunable diffractive elements could find application in various photonic devices. (c) 2013 Optical Society of America

OCIS codes: (050.2770) Gratings; (160.3710) Liquid crystals; (220.1140) Alignment.

http://dx.doi.org/10.1364/OL.38.002342
\end{abstract}

A tunable liquid crystal (LC) grating is one of the most important tools for many of the applications that have been well explored recently [1-4]. The most common approach deals with arrays of parallel electrodes for generating a longitudinal $[\underline{5}, 6]$ or lateral $[\underline{7}, 8]$ periodic electrical field distribution and locally controlling the directors of the LCs and thus the diffraction. The second strategy is to directly pattern the LC alignment layers either by microrubbing $[9,10]$ or by patterned photoalignment [11] and guiding the LC director in different alignment domains. Another approach is to utilize the natural diffracting ability of the chiral LCs [12]. Such gratings are electrically tunable but cannot be tuned optically $[12,13]$.

In recent years some reports dealing with optical tunability of dye-doped organic materials have been written [14]. Confined complex structures such as polymerLC-polymer-slide (PLCPS) gratings containing azo compounds show optical switching times of $0.5 \mathrm{~s}$ at the irradiance of $245 \mathrm{~mW} / \mathrm{cm}^{2}$ and diffraction efficiencies (DEs) up to $85 \%$ [15]. In another report, a $90 \% \mathrm{DE}$ and switching time of $0.2 \mathrm{~s}$ (at the irradiance of $54 \mathrm{~mW} /$ $\mathrm{cm}^{2}$ ) were reported for PLCPS with photosensitive LC [16]. Most of these reports include dye-doped LC for a photopolymerizable mixture for recording the grating. However, azo dyes, because of their chemical instability, are not stable in the LC mixture, and therefore the efficiency of these devices is highly limited and they manifest a rather complex fabrication procedure [17]. Another approach, based on a polydimethylsiloxane periodic microstructure on a glass substrate, separated by a thin LC film, with a DE of $35 \%$ and switching time of $145 \mathrm{~s}$ at the irradiance of $600 \mathrm{~mW} / \mathrm{cm}^{2}$, has been reported [18].

Other than LC, a metamaterial [19] fabricated by encapsulating photochromic solution in nano-holes of polycarbonate has also been proposed for fabricating optically tunable gratings [4]. The interference pattern by the green laser was used to write the grating profile, whereas it can be erases by UV irradiation $[20,21]$. However, the technology is restricted by the large switching time of $\sim 30 \mathrm{~s}$ (at an irradiance of $300 \mathrm{~mW} / \mathrm{cm}^{2}$ ).

Recently, we have disclosed a relatively easier and more reliable method to fabricate electrically tunable gratings, by defining the LC director in alternate alignment domains of alternate planar (PA) and twisted (TN) alignments by means of photoalignment [22]. Moreover, among all of these strategies, patterned photoalignment has been demonstrated to be efficient [11-13].

In this article, we present an optically tunable LC grating based on PA and TN alignment domains. The blue light $(\lambda=450 \mathrm{~nm})$ can be used to tune the diffractive and nondiffractive states. Such gratings can also be erased and rewritten for different grating vectors. Thus, they could find several applications.

The optically tunable and rewritable (OTRW) LC cell consists of two substrates with different aligning materials, one of which is optically passive (insensitive to light exposure) and keeps a fixed easy axis, whereas the other aligning layer is optically active and can change its easy axis after being exposed by polarized light. A sulfonic azo dye (SD1) (Dai-Nippon Ink and Chemicals, Japan) is used as the optically active alignment layer. The exposure of the SD1 layer by the polarized light of wavelength ( $\lambda$ ) $450 \mathrm{~nm}$ provides the alignment in the direction perpendicular to the E-vector of the exposing light, with an almost zero pretilt angle and high azimuthal anchoring energy $\left(A_{\varphi}\right)$. Moreover, the easy axis of the SD1 layer can be changed by another exposure through the polarized light of the same $\lambda$ but a different direction of the E-vector [23]. The rewriteable alignment with the almost zero pretilt angle provides an opportunity to tune the diffraction profile optically. Furthermore, it can be erased and rewritten for any desirable preferences.

The OTRW LC cell has been prepared by coating the $0.5 \%$ solution of SD1 in N,N-dimethylformamide for the optically active substrate. The optically passive layer can be made of any photoinsensitive alignment material; in the present case a $2 \%$ solution of PI 3744 (from Chisso Chemical, Japan) is used. The cell thickness was maintained at $10 \mu \mathrm{m}$. Figure 1 (a) shows the typical cell structure.

First both substrates were treated to provide the PA alignment. After filling the PA cell with LC, the optically active layer (i.e., SD1) of the cell was exposed by polarized light of the same $\lambda$, having the E-vector in the 
perpendicular direction to the previous exposure, through the mask. Therefore, the easy axis of SD1, for the exposed area, reorients orthogonally with respect to the covered region. Figures $1(\mathrm{~b})$ and $1(\mathrm{c})$ show a mask that has been transferred to OTRW cell. Several gratings, including a 1D and a 2D grating, have been prepared, and some of them are shown in Figs. 1(d) and 1(e). In Fig. 1(e), due to beam size expansion, the transferred pattern looks like circular disks rather than squares. Moreover, an interference pattern, diffraction pattern, or pattern generated by a microelectromechanical system can also be used to modify the grating vector of the OTRW LC cell.

The writing and erasing of such gratings are controlled by the tuning of $A_{\varphi}$ by the exposure energy (i.e., the product of irradiance and time). Therefore, $A_{\varphi}$ has been measured for different exposure energy by measuring the effective twist angle [1, 14]. $A_{\varphi}$ for SD1 increases with the exposure energy and saturates to its maximum level (Fig. 2) [23]. Thus, after finite exposure energy, $A_{\varphi}$ memorizes its value and easy axis, which does not change even after removal of the exposing light. The easy axis and value of $A_{\varphi}$ can only be changed by another exposure by the same light source with a different E-vector. Moreover, $A_{\varphi}$ also depends on the SD1 layer thickness; for such applications it has already been optimized to $10 \mathrm{~nm}$ [22-24].

The schematic of the experiments has been shown in a inset of Fig. 2. The blue laser is used as the tuning beam, while the diffraction profile has been studied by green laser $(\lambda=550 \mathrm{~nm})$. Thus, a light flash (controlled by the LC optical shutter) with sufficiently high exposure intensity is enough to write or erase the OTRW gratings. The same setup was also used to study the optical tunability.

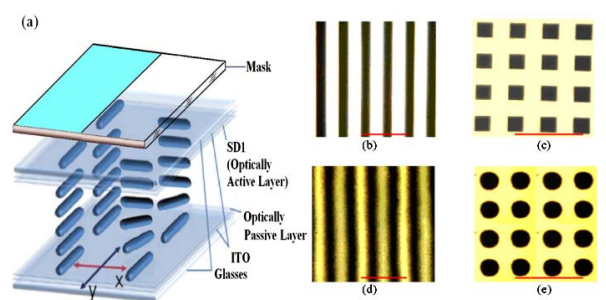

Fig. 1. (a) Principle structure and operation of OTRW LC grating cell; (b), (c) the optical $1 \mathrm{D}$ and $2 \mathrm{D}$ grating masks; (d), (e) the micrographs for the grating LC cell. The marker size is $100 \mu \mathrm{m}$.

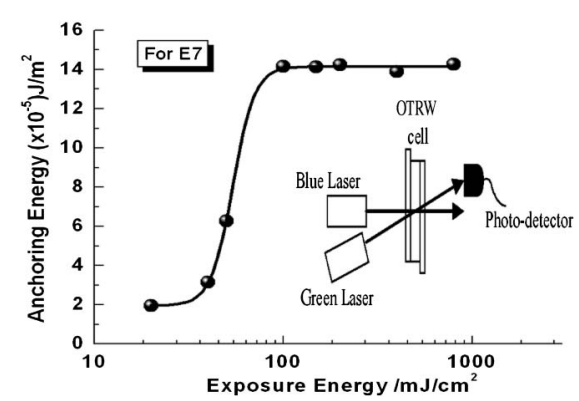

Fig. 2. Anchoring energy dependence of the SD1 layer on the exposure energy at fixed irradiance $\left(500 \mathrm{~mW} / \mathrm{cm}^{2}\right)$. The experimental arrangement for the optical tunability is presented in the inset.
Both 1D and 2D gratings with a pitch of $50 \mu \mathrm{m}$ have been recorded in the OTRW cell and are presented in Figs. 1(d) and 1(e), respectively. Thereafter, the blue laser with a perpendicular E-vector to that of the previous exposure was used to erase the patterns from the OTRW cell. After, the same OTRW cell was rewritten for a different pattern with a period of $25 \mu \mathrm{m}$. The diffraction patterns of the OTRW cell with a $50 \mu \mathrm{m}$ period, erased (no diffraction) and rewritten for a period of $25 \mu \mathrm{m}$, are shown in Figs. 3(a)-3(c), respectively. Thereafter, the pattern was erased again and rewritten for the period of $25 \mu \mathrm{m}$ in the direction perpendicular to the one in Fig. 3(c) [Fig. 3(d)]. In the next step, a 2D grating with a pitch of $50 \mu \mathrm{m}$ was recorded [Fig. 3(e)]. The pitch of the structure can be reduced up to $10 \mu \mathrm{m}$ [24].

For optical tunability (inset, Fig. 2), the writing and erasing speed are important parameters. As stated earlier, the exposure energy is responsible for both the writing and erasing processes. Thus, both of these times are the same and can be termed the response time $(\tau)$, which is defined as the time elapsed in changing the transmittance of the first-order beam from $10 \%$ to $90 \%$ or vice versa. The first-order transmittances for three different LCs (5CB, E7, and TN403) have been plotted against the exposure energy in Fig. 4. The transmittance increases with the exposure energy and then saturates to the maximum value. This maximum value corresponds to the twist angle, $\sim 90^{\circ}$ for the $\mathrm{TN}$ domains, and does not change at a higher exposure energy. This figure also reveals that the OTRW grating can be tuned more rapidly by a higher exposure intensity.

In addition to a higher exposure energy, LC parameters are also very important. The exposing light creates TN

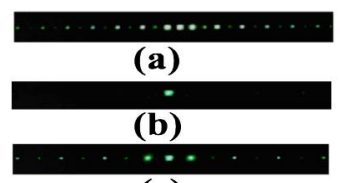

(c)

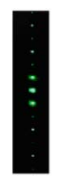

(d)

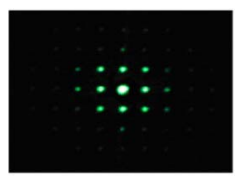

(e)
Fig. 3. Diffraction profiles of the OTRW gratings prepared in the same cell: (a) $1 \mathrm{D}$ grating, period $=50 \mu \mathrm{m}$, (b) no diffraction structure, (c) 1D grating, period $=25 \mu \mathrm{m}$, (d) 1D grating, period $=25 \mu \mathrm{m}$ in the perpendicular direction to the previous structure, and (e) 2D grating, period $=50 \mu \mathrm{m}$.

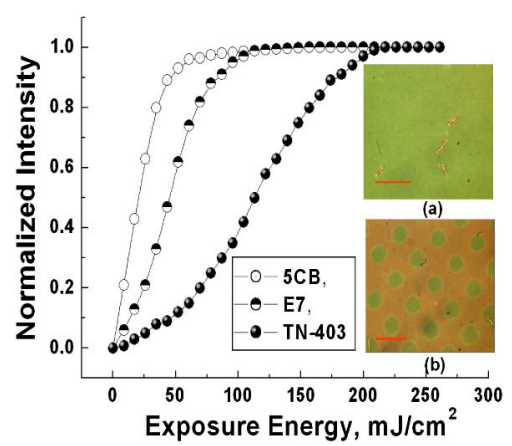

Fig. 4. Effective transmittance dependence on exposure energy, at fixed irradiance $\left(500 \mathrm{~mW} / \mathrm{cm}^{2}\right)$ of the writing beam, for LCs with different $K_{22}$. Insets (a) and (b) represent the optical microphotograph for the cell before and after writing of the grating, respectively, under a crossed polarizer. The marker size is $50 \mu \mathrm{m}$. 
domains and consequently the grating, and therefore the dynamics of the proposed grating critically relates to the effective twist angle $(\varphi)$. Furthermore, the $\varphi$ is related to $A_{\varphi}$ of the SD1 layer as $A_{\varphi}=2 \varphi K_{22} / d \sin (\Phi-\varphi)[\underline{1}, \underline{14}]$, where $\Phi, K_{22}$, and $d$ are the desired twist angle, twist elastic constant of the LCs, and cell gap, respectively. As stated before, $A_{\varphi}$ is mainly dependent on the exposure energy. Moreover, $A_{\varphi}$ and $\varphi$ increase at higher exposure energy. Thus before saturation, the fixed exposure energy offers a fixed value of $A_{\varphi}$ and thus a limited $\varphi$. Likewise, it is also clear that the LCs with smaller $K_{22}$ will produce higher $\varphi$ for the same exposure energy, and therefore the switching speed for the LCs with smaller $K_{22}$ is expected to be faster. Hence in Fig. 4 , the LC with the smallest $K_{22}$, i.e., $5 \mathrm{CB}$, shows a higher transmittance at less exposure energy. In other words, $5 \mathrm{CB}$, because of the smaller $K_{22}$, is faster than E7 and TN403. Thus, through the selection of a sufficiently high exposure intensity and proper LC with smaller $K_{22}$, one can optimize the $\tau$. Here we have achieved a $\tau$ of $100 \mathrm{~ms}$ by the laser of intensity $500 \mathrm{~mW} / \mathrm{cm}^{2}$, which can be improved further.

The first-order DEs for 1D and 2D gratings (computed from Fig. 3) are $34 \%$ and $40 \%$, respectively. However, the DE of such gratings can be improved further by optimizing the cell and LC parameters [13].

In summary, an OTRW LC grating has been proposed that is based on alternate TN and PA domains. The proposed OTWR grating can be optically tuned, erased, and rewritten with the polarized light. The achieved firstorder $\mathrm{DE}$ in the present case is $34 \%$ and $40 \%$ for a $1 \mathrm{D}$ and a 2D grating, respectively, which can be improved further. The response time of such OTRW gratings depends on the exposure intensity and LC parameters. Therefore, the proposed OTRW grating has a high resolution and fast switching speed ( $100 \mathrm{~ms})$ at considerably less exposure energy than the existing technologies. Thus, it could find application in a wide range of photonic and adaptive optical elements and other optical devices [ $\underline{10}-14]$.

This work is supported by HKUST grants CERG 612409, CERG 612310, and RGC 614410.

\section{References}

1. V. G. Chigrinov, Liquid Crystal Devices: Physics and Applications (Artech-House, 1999).
2. J. Chen, P. J. Bos, H. Vithana, and D. L. Johnson, Appl. Phys. Lett. 67, 2588 (1995).

3. W. Y. Wu and A. Y. G. Fuh, Jpn. J. Appl. Phys. 46, 6761 (2007).

4. S. Y. Huang, S. T. Wu, and A. Y. G. Fuh, Appl. Phys. Lett. 88, 041104 (2006).

5. M. Bouvier and T. Scharf, Opt. Eng. 39, 2129 (2000).

6. L. L. Gu, X. N. Chen, W. Jiang, B. Howley, and R. T. Chen, Appl. Phys. Lett. 87, 201106 (2005).

7. R. G. Lindquist, J. H. Kulick, G. P. Nordin, J. M. Jarem, S. T. Kowel, M. Friends, and T. M. Leslie, Opt. Lett. 19, 670 (1994).

8. J. Yan, Y. Li, and S. T. Wu, Opt. Lett. 36, 1404 (2011).

9. B. Wen, R. G. Petschek, and C. Rosenblatt, Appl. Opt. 41, 1246 (2002).

10. V. Kapoustine, A. Kazakevitch, V. So, and R. Tam, Opt. Commun. 266, 1 (2006).

11. W. Hu, A. K. Srivastava, X. Liang, X. W. Lin, J. Sun, G. Zhu, V. Chigrinov, and Y. Q. Lu, Appl. Phys. Lett. 100, 111116 (2012).

12. A. K. Srivastava, E. P. Pozhidaev, V.G. Chigrinov, and R. Manohar, Appl. Phys. 99, 201106 (2011).

13. F. Fan, A. K. Srivastava, V. G. Chigrinov, and H. S. Kowk, Appl. Phys. Lett. 100, 111105 (2012).

14. V. G. Chigrinov, V. M. Kozenkov, and H. S. Kwok, Photoalignment of Liquid Crystalline Materials: Physics and Applications (Wiley, 2008).

15. L. De Sio, A. Veltri, C. Umeton, S. Serak, and N. Tabiryan, Appl. Phys. Lett. 93, 181115 (2008).

16. L. De Sio, S. Serak, N. Tabiryan, S. Ferjani, A. Veltri, and C. Umeton, Adv. Mater. 22, 2316 (2010).

17. A. K. Srivastava and R. Manohar, Liq. Cryst. 38, 183 (2011).

18. L. De Sio, J. G. Cuennet, A. E. Vasdekis, and D. Psaltis, Appl. Phys. Lett. 96, 131112 (2010).

19. M. Saito, Y. Tsubokura, N. Ota, and A. Fujiuchi, Appl. Phys. Lett. 91, 061114 (2007).

20. I. C. Khoo, S. Slussarenko, B. D. Guenther, Min-Yi Shih, P. Chen, and W. V. Wood, Opt. Lett. 23, 253 (1998).

21. D. E. Lucchetta, F. Vita, and F. Simoni, Appl. Phys. Lett. 97, 231112 (2010).

22. W. Hu, A. Srivastava, F. Xu, J. Sun, X. W. Lin, H. Q. Cui, V. Chigrinov, and Y. Q. Lu, Opt. Express 20, 5384 (2012).

23. A. D. Kiselev, V. G. Chigrinov, and H. S. Kwok, Phys. Rev. E 80, 011706 (2009).

24. A. K. Srivastava, Wei Hu, V. G. Chigrinov, A. D. Kiselev, and Yan-Qing Lu, Appl. Phys. Lett. 101, 031112 (2012). 